Rabaska

Revue d'ethnologie de l'Amérique française

\title{
Francine Brunel-Reeves (1933-2018)
}

\section{Robert Bouthillier}

Volume 16, 2018

URI : https://id.erudit.org/iderudit/1051334ar

DOI : https://doi.org/10.7202/1051334ar

Aller au sommaire du numéro

Éditeur(s)

Société québécoise d'ethnologie

ISSN

1703-7433 (imprimé)

1916-7350 (numérique)

Découvrir la revue

Citer ce document

Bouthillier, R. (2018). Francine Brunel-Reeves (1933-2018). Rabaska, 16, 202-205. https://doi.org/10.7202/1051334ar d'utilisation que vous pouvez consulter en ligne.

https://apropos.erudit.org/fr/usagers/politique-dutilisation/ 


\section{Nécrologie}

\section{Francine Brunel-Reeves (1933-2018)}

En matière de chanson et de musique traditionnelles, peu de gens auront réussi la synthèse entre l'univers de la recherche et celui de la pratique comme l'a fait depuis plus de 40 ans Francine Brunel-Reeves, chanteuse, câlleuse et ethnologue autodidacte, qui nous a quittés le 2 février dernier à l'âge de 84 ans. Il fut un temps où on était soit chercheur, soit chanteur (ou conteur, ou danseur, ou violoneux...), mais à peu près jamais les deux à la fois. Et quand passerelles il y a eu - grosso modo depuis les années 1980 - les praticiens à double casquette « labellisés » ethnologues ont pour la plupart légitimé leur appartenance à la communauté scientifique grâce à l'obtention d'un diplôme décerné par une institution universitaire reconnue.

Francine Brunel a suivi un tout autre

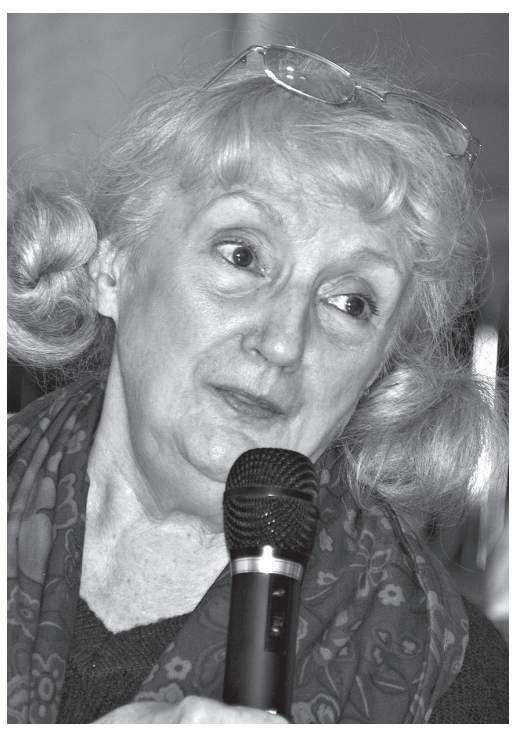

Francine Brunel-Reeves en train de chanter dans une veillée de chant à Mont-Rolland, Québec Photo : Jean Richard, novembre 2012 chemin. Née à Montréal en 1933 dans une famille culturellement douée (père universitaire en sciences naturelles), scolarisée jusqu'au cours commercial - ce qui, dans le Québec des années 1950, était déjà remarquable quand on était une jeune fille - dans une école tenue par les sœurs de Jésus-Marie, qui lui auront appris, en plus du secrétariat, à jouer du piano..., mariée en 1955 à une future sommité de l'astrophysique dont elle a emprunté pour un temps le patronyme ${ }^{1}$, elle semblait destinée à

1. Francine a commencé sa carrière en portant le seul nom de Reeves. Après son divorce, elle n'a pas voulu s'en départir parce que c'était sous ce nom qu'elle était connue du public. Ce n'est que 
être une mère de famille «normale »-quatre enfants en six ans - comme il était d'usage dans le Québec d'avant la Révolution tranquille, alors que la femme devait suivre son mari... Ce qu'elle a fait d'ailleurs, au début, et grand bien lui fit, car elle découvrira alors des mondes d'histoire et de culture que peu de Québécoises de l'époque ont eu la chance de fréquenter d'aussi près : une période d'alternance (1956-1963) entre Ithaca, New-York et Montréal, puis, le grand saut en Europe, en Belgique d'abord (1964) et à Paris à partir de 1965. Elle y restera 26 ans, jusqu'à son retour au Québec en 1991².

À l'exception d'un bref contact au début des années 1950 avec la danse folklorique en milieu de loisir (troupe Les Folkloristes du Québec sous la direction de Simonne Voyer), rien ne laissait alors présager la passion future de Francine pour la tradition orale. Elle a d'abord commencé, au début des années 1970, par s'investir totalement dans l'univers des chanteurs-auteurscompositeurs-interprètes « rive gauche » et c'est le hasard qui l'a amenée à proposer en 1975 l'animation d'un «bal folk » québécois sur la base de ses réminiscences adolescentes. Sans abandonner l'univers des AcI, le succès rencontré l'a encouragée à continuer à animer des soirées de danses québécoises, travaillant d'abord sur la base de musique enregistrée, puis avec trois équipes successives de musiciens traditionnels revivalistes. Elle aurait pu s'en tenir là et limiter son regard et sa pratique au monde du folk. Mais étant parfaitement consciente des limites de son savoir de dilettante, dotée d'une personnalité aussi curieuse qu'enthousiaste et cherchant toujours à aller plus loin sur les chemins de la connaissance, elle a voulu explorer d'autres sources que celles auxquelles s'abreuvaient la grande majorité de ses camarades revivalistes. Et elle a croisé son « chemin de Damas » en 1982, à l'occasion d'un long périple au Québec qui lui a permis de rencontrer des dizaines de musiciens et de chanteurs traditionnels de l'ancienne génération encore actifs - ceux qu'on appelle aujourd'hui des " porteurs de tradition » et de prendre conscience du même coup de la richesse du terrain et de l'apport irremplaçable de la démarche ethnographique pour nourrir à la fois sa pratique et son savoir.

À partir de ce jour, les choses ne seront plus jamais les mêmes, et Francine n'envisagera plus la tradition de la même façon. Pour gagner sa vie, elle a continué d'animer des bals québécois jusqu'à son retour au Québec en 1991,

\footnotetext{
beaucoup plus tard, une fois revenue au Québec, qu'elle a accolé son propre patronyme au « nom de scène » qu'elle avait porté pendant vingt ans.

2. Je résume à dessein, des informations beaucoup plus détaillées sur le parcours de Francine ayant déjà été publiées dans cette même revue, sous la plume de Marc Bolduc : «Francine BrunelReeves : portrait d'une chercheuse atypique ou quand la pratique appelle la recherche... », Rabaska, vol. 6, 2008, p. 79-92. On pourra aussi lire avec bonheur l'interview conséquente réalisée par Philippe Krumm, «Francine Brunel-Reeves. Une vie en musique », publiée dans Trad Magazine, n 161, mai/ juin 2015 .
} 
mais elle s'est surtout passionnée pour les traditions orales et musicales dans leur ensemble, élargissant au contact de chercheurs reconnus - Jean-Michel Guilcher, Jean-Loïc Le Quellec, Georges Delarue, Brigitte Charnier, et quelques autres... - le spectre de sa curiosité première pour la danse et la musique vers la chanson, les récits, l'histoire, la mythologie, la linguistique. Elle a raconté dans une interview comment sa passion pour la complainte $L a$ Blanche Biche a commencé ${ }^{3}$, au point qu'elle a même (brièvement) songé à en faire un sujet de thèse. Qu'elle n'ait pas franchi le pas universitaire n'a aucune importance : elle est de toute façon la personne qui a accumulé la plus importante documentation connue sur cette chanson devenue quasi mythique grâce à elle, traquant ses motifs narratifs et étymologiques à travers le temps et l'espace jusqu'en Scandinavie et en Inde, au point qu'on l'a parfois affectueusement surnommée « Madame Blanche Biche».

Mais La Blanche Biche est aussi l'arbre qui cache la forêt et ses recherches ne s'y sont pas arrêtées, loin d'en faut. Dès les années 1970, donc bien avant le voyage québécois de 1982, Francine enregistrait presque tout ce qu'elle croisait sur sa route de quête musicale : spectacles, concerts, festivals, veillées..., accumulant les traces sonores laissées aussi bien par des chanteurs et musiciens revivalistes que par des interprètes plus enracinés. Elle ne cherchait pas tant « l'authenticité », indéfinissable par ailleurs, que les traces de vie de cette musique dans tous ses états dont elle engrangeait le son et l'image pour nourrir sa propre mémoire. Au fil des ans, et l'ethnographie plus classique étant venue élargir le propos et donner une autre dimension à ses premiers enregistrements, Francine a constitué un fonds aussi impressionnant - plusieurs centaines de supports physiques, bandes, cassettes et vidéos qu'unique par le regard à la fois non sélectif et composite qu'il permet de jeter sur l'ensemble du phénomène des chansons et musiques traditionnelles telles qu'elles étaient et sont pratiquées dans les contextes de la culture contemporaine. C'est aussi par dizaines qu'elle a constitué des dossiers documentaires sur un thème général ou une chanson spécifique, selon les coups de cœur qu'elle éprouvait en découvrant une version qui contenait un motif qui l'interpellait. Par exemple (pour n'en proposer qu'un), pourquoi le « pauvre soldat qui revient de guerre tout mal chaussé tout mal vêtu »a-t-il, dans plusieurs versions, « un pied chaussé et l'autre nu $»^{4}$, dès lors que le diable, dans une toute autre chanson, apparaît lui aussi « un pied chaussé et l'autre nu » avant d'être mis en fuite par une aspersion d'eau bénite ${ }^{5}$ ? C'est

3. Cf. l'interview de Francine dans Trad Magazine, op. cit., p. 28, $3^{\mathrm{e}}$ colonne.

4. Laforte II.I-05 - Le Retour du mari soldat : trois enfants. Voir par exemple la version publiée par Dominique Gauthier et Roger Matton dans Chansons de Shippagan, Québec, Put, 1975, p. 9.

5. Laforte II.B-42 - Le Docteur qui vend son âme au diable. Texte critique établi par Luc Lacourcière et publié dans Jean Du Berger, Introduction à la littérature orale (documentation); Québec, PuL,1971, document 211/04. 
le genre de questions pour lesquelles Francine se passionnait et elle aurait remué ciel et terre, en quête de toutes les versions possibles et de toutes les sources qui en parlaient, pour en découvrir l'origine ou, à défaut, le sens.

Ces dossiers sont pour partie «physiques »- articles, photocopies diverses, notes manuscrites ou tapuscrites...-mais aussi, pour une grande part, dématérialisés. Son centre de documentation personnel, au-delà des disques vinyle, des cassettes et des livres qui donnaient à son appartement montréalais des allures de caverne d'Ali Baba, était surtout contenu dans le disque dur de son Mac, sur lequel elle engrangeait à la fois documents sonores numérisés et versions transcrites par ses soins ou réécrites sous forme de documents textes informatisés. À la différence des chercheurs disparus qui l'ont précédée et qui ont essentiellement constitué des « fonds papier », l'héritage intellectuel de Francine tient aussi dans les dossiers numériques qu'elle a élaborés au fil du temps, ce qui constituera d'ailleurs une problématique inédite pour les documentalistes qui auront la tâche de faire tri entre ce qui relève d'un fonds Brunel-Reeves et ce qui a été emprunté à des collections préexistantes. En effet, y sont fusionnés aussi bien des documents qu'elle a recueillis dans ses enquêtes que des documents rassemblés à partir des collectes et des fonds où elle s'est nourrie, non pas avec des intentions d'appropriation personnelle - Francine se faisait un point d'honneur d'indiquer toutes les références de chacun des documents qu'elle versait dans un dossier - mais dans un but de documentation et de diffusion des connaissances.

Car, si elle était avide de savoir, si elle s'enthousiasmait pour les chansons, elle débordait d'admiration et d'amour pour les porteurs de tradition qu'elle avait rencontrés et côtoyés pendant une trentaine d'années et dont elle parlait magnifiquement ${ }^{6}$. Et elle ne désirait au final qu'une chose : transmettre cette connaissance et cet enthousiasme à celles et ceux qui n'avaient pas eu la chance ou l'occasion de rencontrer la tradition et ses porteurs dans les mêmes conditions qu'elle. Et au-delà de cette volonté de transmettre de l'information, elle voulait aussi amener les gens qui se découvraient un intérêt pour la chanson à se réapproprier la matière chantée. Dès son retour au Québec en 1991, Francine s'est investie dans la vie associative (SPDTQ, CQPV, SQE...) et a joué entre autres un rôle catalytique de premier plan dans la réactualisation des « veillées de chant »-avec l'intention d'en faire non pas un objet de musée et d'archives, mais un objet de pratique d'ici et maintenant, inscrit bien sûr dans une histoire et une continuité, mais pouvant aussi se projeter dans un avenir si tant est qu'il reste non pas une, mais des voix pour en assurer le passage.

RoBert Bouthillier

Caen, juillet 2018

6. Par exemple, dans le très beau film documentaire Tant qu'il reste une voix, réalisé en 2008 par Jean-Nicolas Orhon sur le parcours de Francine Brunel-Reeves (DVD, distribution Netima, 52 min.). 